\title{
Uma revisão sobre o acolhimento na atenção à saúde de adolescentes
}

\author{
A review on User Embracement in adolescent health care \\ Una revisión sobre la acogida de la atención de la salud de los adolescentes
}

Recebido: 13/02/2021 | Revisado: 20/02/2021 | Aceito: 22/02/2021 | Publicado: 28/02/2021

\author{
Laila Felix de Melo \\ ORCID: https://orcid.org/0000-0003-4212-6376 \\ Universidade Federal de Minas Gerais, Brasil \\ E-mail: lailafelixmelo@gmail.com \\ Pedro Augusto Pereira dos Santos \\ ORCID: https://orcid.org/0000-0001-5932-8252 \\ Universidade Federal de Minas Gerais, Brasil \\ E-mail: pedropsantos.95@gmail.com \\ João Henrique Lara do Amaral \\ ORCID: https://orcid.org/0000-0001-6900-7559 \\ Universidade Federal de Minas Gerais, Brasil \\ E-mail: jhlamaral@ufmg.br \\ Najara Barbosa da Rocha \\ ORCID: https://orcid.org/0000-0003-3366-0032 \\ Universidade Federal de Minas Gerais, Brasil \\ E-mail: najaraufmg@ufmg.br
}

\begin{abstract}
Resumo
Objetivo: Realizar uma revisão de literatura sobre acolhimento e atenção à saúde de adolescentes. Metodologia: Tratase de uma revisão de literatura, com uma busca sistematizada. As bases de dados consultadas foram Biblioteca Virtual em Saúde (BVS) e Pubmed e os critérios de inclusão: estudos sobre o tema; trabalhos completos e disponíveis; idiomas português, inglês ou espanhol; trabalhos publicados à partir de 2004; todos tipos de estudo, exceto revisão. O conteúdo dos trabalhos foi lido em profundidade e definidas as categorias de análise. Resultados: A busca pelos descritores resultou em 713 estudos, que após a leitura do título, foram reduzidos a 49. Após leitura dos resumos foram selecionados 15 trabalhos para leitura completa. Destes, 9 se encaixaram aos critérios do estudo. Foi encontrada uma baixa procura dos adolescentes pelos serviços de saúde. Foram identificadas condições nos serviços de saúde que interferem na atenção à saúde dos adolescentes. Essas condições foram classificadas como dimensão macro, meso e micro. A dimensão macro se refere a condições do modelo de atenção. A dimensão meso abrange as condições estruturais da atenção ao adolescente e capacitação dos profissionais de saúde. Já a dimensão micro diz respeito às relações interpessoais entre adolescentes e profissionais de saúde. Conclusão: Os resultados deste estudo sugerem que o atendimento à saúde do adolescente exige iniciativas que respondam suas especificidades e, que o acolhimento é fundamental para aproximar o adolescente desse cuidado, pois humaniza, cria vínculo, respeito pela individualidade e autonomia.
\end{abstract}

Palavras-chave: Acolhimento; Adolescente; Saúde; Acesso aos serviços de saúde; Atenção primária em saúde.

\begin{abstract}
Objective: To review the literature on User Embracement and adolescent health care. Methodology: This is a literature review, with a systematized search. The databases consulted were Virtual Health Library (VHL) and Pubmed and inclusion criteria: studies on the subject; complete and available works; languages Portuguese, English or Spanish; papers published from 2004; all types of study, except review. The content of the papers was read in depth and the categories of analysis were defined. Results: The search for the descriptors resulted in 713 studies, which after reading the title, were reduced to 49. After reading the abstracts, 15 papers were selected for full reading. Of these, 9 met the study criteria. A low demand of adolescents for health services was found. Conditions were identified in health services that interfere in the health care of adolescents. These conditions were classified as macro, meso and micro dimension. The macro dimension refers to the conditions of the care model. The meso dimension covers the structural conditions of adolescent care and training of health professionals. The micro dimension concerns interpersonal relationships between adolescents and health professionals. Conclusion: The results of this study suggest that adolescent health care requires initiatives that respond to its specificities and User Embracement is fundamental to bring adolescents closer to care, as it humanizes, creates bonding, respect for individuality and autonomy.
\end{abstract}

Keywords: User embracement; Adolescent; Health; Health services accessibility; Primary health care. 


\begin{abstract}
Resumen
Objetivo: Llevar a cabo una revisión de la literatura sobre el miedo y la atención médica de los adolescentes. Metodología: Esta es una revisión de la literatura, con una búsqueda sistematizada. Las bases de datos consultadas fueron Virtual Health Library (VHL) y Pubmed y criterios de inclusión: estudios sobre el tema; obras completas y disponibles; idiomas portugués, inglés o español; artículos publicados a partir de 2004; todo tipo de estudio, excepto la revisión. El contenido de los documentos se leyó en profundidad y se definieron las categorías de análisis. Resultados: La búsqueda de los descriptores dio lugar a 713 estudios, que después de leer el título, se redujeron a 49. Después de leer los resúmenes, se seleccionaron 15 artículos para su lectura completa. De ellos, 9 cumplieron con los criterios de estudio. Se encontró una baja demanda de servicios de salud para los adolescentes. Se identificaron condiciones en los servicios de salud que interfieren en la atención médica de los adolescentes. Estas condiciones se clasificaron como macro, meso y micro dimensión. La dimensión macro hace referencia a las condiciones del modelo de cuidado. La dimensión meso abarca las condiciones estructurales de la atención a los adolescentes y la formación de los profesionales de la salud. La micro dimensión se refiere a las relaciones interpersonales entre adolescentes y profesionales de la salud. Conclusion: Los resultados de este estudio sugieren que la atención de la salud de los adolescentes requiere iniciativas que respondan a sus especificidades y que la acogida es fundamental para acercar a los adolescentes a la atención, porque humaniza, crea vinculación, respeto a la individualidad y autonomía.

Palabras clave: Acogimiento; Adolescente; Salud; Accesibilidad a los servicios de salud; Atención primaria de salud.
\end{abstract}

\title{
1. Introdução
}

A adolescência, definida como o período dos 10-19 anos de idade, é o intervalo de transição da infância para vida adulta, caracterizado pelo crescimento e desenvolvimento físico, mental, emocional e social. Em geral, é um ciclo da vida que pode ser acompanhado de "desafios", como a adaptação às mudanças fisiológicas e anatômicas associadas à puberdade. A expectativa pela independência afetiva e financeira com relação à família dá lugar aos relacionamentos interpessoais com os pares. Ainda, ocorre o desenvolvimento da identidade individual e sexual, aquisição de um senso de comunidade, desenvolvimento da capacidade de trabalho e atividades de lazer que sejam relevantes tanto individual quanto nas relações com a comunidade (WHO, 1986).

Os adolescentes quando pacientes requerem novos modos de produzir saúde uma vez que os agravos em saúde podem decorrer de hábitos e comportamentos. Também podem ocorrer condições de alta vulnerabilidade principalmente em relação ao contexto social. Entre os adolescentes pode ocorrer: gravidez precoce, início de uso de drogas lícitas e ilícitas, violência nas suas diferentes formas e doenças sexualmente transmissíveis, entre outras. A adolescência é uma fase da vida de grande exposição a vários fatores de risco à saúde e muitos dos hábitos adquiridos são levados para a vida adulta com consequências para a saúde. Nesse sentido, as especificidades da vida do adolescente devem orientar o acolhimento e a atenção integral dessa população que busca a atenção básica em saúde (Brasil, 2017).

O acolhimento no Sistema único de Saúde (SUS) é parte da Política Nacional de Humanização 2004. Com essa política valoriza-se a escuta e o protagonismo do indivíduo enquanto detentor potencial da determinação da própria saúde (Brasil et al., 2012).

O Acolhimento, enquanto elemento constitutivo da PNH (Brasil, 2004), é "uma postura ética no que se refere ao compromisso com o reconhecimento do outro, na atitude de acolhê-lo em suas diferenças, suas dores, suas alegrias, seus modos de viver, sentir e estar na vida; postura estética porque traz para as relações e os encontros do dia-a-dia a invenção de estratégias que contribuem para a dignificação da vida e do viver e, assim, para a construção de nossa própria humanidade e postura política porque implica o compromisso coletivo de envolver-se neste "estar com", potencializando protagonismos e vida nos diferentes encontros".

O Acolhimento, enquanto processo nos serviços de atenção à saúde, não se restringe à recepção do paciente, mas inclui o diagnóstico, encaminhamento e acompanhamento do paciente até a resolução integral das necessidades de saúde. O Acolhimento deve considerar o direito aos cuidados de saúde, o protagonismo dos sujeitos e ser solidário, universal e responsável pela resolução dos problemas de saúde do paciente (Hennington, 2005). Nesse momento, a troca de saberes entre o 
profissional de saúde e o indivíduo é fundamental para fortalecer a interação entre esses sujeitos (Emmi et al., 2016).

No SUS o Acolhimento é uma tecnologia a ser usada na organização dos serviços e a sua implementação tem como resultado não só o aprofundamento da interação entre profissionais/usuários/gestores, como também na resolutividade de casos e eficiência no atendimento das necessidades de saúde (Brasil, 2010).

O Acolhimento na atenção à saúde ao adolescente é um tema com potencial retorno para o cuidado em saúde, porém ainda é pouco estudado. Por isso, este trabalho objetivou avaliar, por meio de uma busca sistematizada e revisão da literatura, os estudos disponíveis para compreender o processo de acolhimento ao adolescente e o acesso aos cuidados em saúde dessa população.

\section{Metodologia}

Trata-se de uma revisão de literatura, busca bibliográfica sistematizada, realizada por dois pesquisadores independentes (LFM e PAPS). Esta revisão inclui aspectos de uma revisão sistemática, como uma busca na literatura com critérios bem definidos e realizada de forma sistematizada, no entanto, sem ser classificada como tal (Grant \& Booth, 2009). O artigo de revisão é um tipo de pesquisa que utiliza de fontes de informações bibliográficas para obtenção de resultados de pesquisas de outros autores, com o objetivo de fundamentar teoricamente um determinado objetivo (Rother, 2007.

Para esta revisão foram percorridas as seguintes etapas: identificação do tema e seleção da questão de pesquisa; estabelecimento de critérios de inclusão e exclusão; identificação dos estudos; categorização dos estudos selecionados; análise e interpretação dos resultados; e apresentação e síntese do conhecimento.

Essa revisão procurou responder a seguinte pergunta: "o que há na literatura sobre o acolhimento e acesso na atenção à saúde de adolescentes?".

Para isso, buscas foram realizadas, em maio de 2020, com os descritores: adolescentes, acolhimento, saúde e acessibilidade aos serviços de saúde com utilização dos operadores boleanos AND e OR nas bases de dados: Biblioteca Virtual em Saúde - BVS (que inclui a Literatura Latino-Americana e do Caribe em Ciências da Saúde - LILACS) e do MedLine (PubMed). Essas bases foram escolhidas por serem abrangentes, confiáveis e incluir estudos internacionais.

Os critérios de inclusão dos estudos foram: estudos compatíveis com o tema; trabalhos completos e disponíveis; idiomas português, inglês ou espanhol; todos os tipos de estudos, exceto revisões; sem recorte territorial; estudos publicados a partir do ano de 2004 até o ano de 2020. Esse período foi definido por ser posterior à implantação da Política Nacional de Humanização no Brasil (Brasil, 2003). Foram definidos como critérios de exclusão: estudos que divergiam da temática e a ausência de texto completo.

Os estudos foram levantados em duas fases. Primeira, foram selecionados artigos pelos títulos e em momento posterior, os resumos. Segunda, os textos completos dos artigos selecionados foram obtidos e identificados pela leitura aqueles que respondiam aos objetivos do estudo. As etapas foram realizadas de forma independente pelos dois pesquisadores e, em momento posterior obtido o consenso. Em caso de discordância, esta foi sanada por um terceiro pesquisador (NBR).

Os artigos incluídos no estudo tiveram seus textos completos lidos e as seguintes informações foram transcritas para uma planilha em formato Microsoft Excel: nome do artigo, autor, ano de publicação e local, metodologia e estratégia de coleta de dados, objetivo do estudo e principais achados. O conteúdo dos trabalhos foi lido em profundidade e definidas categorias de análise.

\section{Resultados}

O Fluxograma abaixo apresenta o processo de seleção dos nove trabalhos que foram incluídos no estudo. 
Figura 1: Fluxograma da identificação, inclusão e exclusão dos artigos.

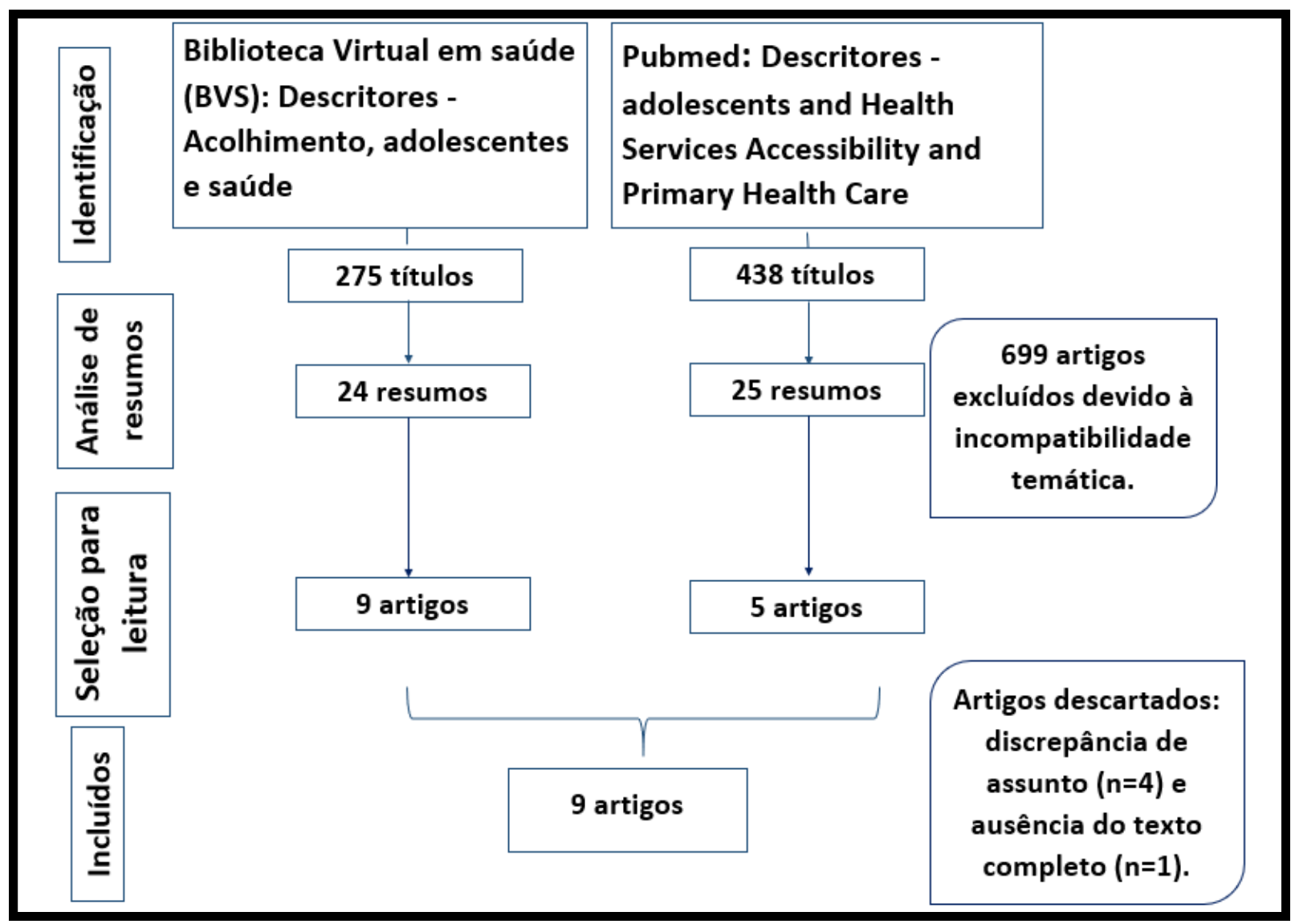

Obs. $\mathrm{n}=$ número. Fonte: Autores.

Inicialmente, 275 artigos foram identificados na BVS e 438 na Pubmed, por meio da busca por descritores. Desses, 699 títulos foram excluídos por não se encaixarem nos critérios de inclusão. Foram analisados 24 resumos da BVS e 25 da Pubmed, e deles, 14 artigos foram selecionados para leitura do texto completo. Após leitura e análise do texto completo, 1 artigo foi descartado por ser uma revisão de literatura, 3 por não abordar o assunto estudado e 1 pela falta do texto completo. Por fim, 9 artigos foram incluídos no estudo (Figura 1).

A Tabela 1 relaciona os dados extraídos dos 9 artigos selecionados. Foram analisados 5 estudos realizados no Brasil, 2 estudos no continente africano, 1 no Reino Unido, e 1 no EUA. 
Tabela 1 - Síntese dos estudos sobre acolhimento e atenção à saúde aos adolescentes.

\begin{tabular}{|c|c|c|c|}
\hline $\begin{array}{c}\text { Título do artigo e nome do(s) } \\
\text { autor(es), ano }\end{array}$ & Objetivo & $\begin{array}{c}\text { Estratégia de coleta de dados e } \\
\text { metodologia }\end{array}$ & Principais achados \\
\hline $\begin{array}{l}\text { Acolhimento coletivo como espaço de } \\
\text { cuidado: uma análise das demandas em } \\
\text { saúde mental na adolescência } \\
\text { Fossi and Reinheimer (2019), Brasil }\end{array}$ & $\begin{array}{l}\text { Analisar as demandas relatadas pelos } \\
\text { pais de adolescentes no acolhimento de } \\
\text { adolescentes em um centro de atenção } \\
\text { psicossocial (CAPS) }\end{array}$ & $\begin{array}{l}\text { Análise do registro escrito de } \\
\text { acolhimentos de pais de adolescentes } \\
\text { que buscaram o CAPS } \\
\text { Período de outubro de } 2014 \text { a fevereiro } \\
\text { de } 2015\end{array}$ & $\begin{array}{l}\text { Os pais foram os que buscaram o atendimento dos } \\
\text { adolescentes } \\
\text { A escuta inicial era realizada com os pais, enão dos } \\
\text { adolescentes, para repassar algumas especificidades do serviço } \\
\text { O estudo mostrou a importância de uma relação horizontal, } \\
\text { com diálogos responsáveis/paciente e profissional, respeitando } \\
\text { sempre a individualidade do adolescente }\end{array}$ \\
\hline $\begin{array}{l}\text { Gestão do cuidado à criança/adolescente } \\
\text { com doença crônica: (des)articulação da } \\
\text { rede e fragmentação das ações } \\
\text { Silva et al. (2020), Brasil }\end{array}$ & $\begin{array}{l}\text { Analisar a gestão do cuidado à criança } \\
\text { e ao adolescente com doença crônica na } \\
\text { estratégia de saúde da família }\end{array}$ & $\begin{array}{l}\text { Pesquisa de natureza qualitativa } \\
\text { exploratória descritiva, realização de } \\
\text { entrevista semiestruturada com } \\
\text { profissionais de saúde em } 5 \text { Unidades de } \\
\text { Saúde da Família (USFs) sorteadas do } \\
\text { munícipio de João Pessoa-PB, com a } \\
\text { análise do discurso } \\
\text { Período de } 2017 \text { a } 2018\end{array}$ & $\begin{array}{l}\text { O acolhimento foi necessário para criar vínculo e confiança } \\
\text { entre o profissional de saúde e paciente } \\
\text { Existe uma grande dificuldade de conciliar o modelo } \\
\text { biomédico com um modelo humanizado de atendimento, } \\
\text { assim, não somente os procedimentos técnicos devem ser } \\
\text { realizados, mas também abordagem integral, singular e } \\
\text { respeitosa } \\
\text { Problemas encontrados: Desresponsabilização no } \\
\text { acompanhamento no território; Falta de contra referência; } \\
\text { Burocratização; Fragilidade no acompanhamento longitudinal } \\
\text { e Fragmentação da rede de atenção do cuidado }\end{array}$ \\
\hline
\end{tabular}




\begin{tabular}{|c|c|c|c|}
\hline $\begin{array}{c}\text { Cuidado ao adolescente na atenção básica: } \\
\text { necessidades dos usuários e sua relação } \\
\text { com o serviço } \\
\text { Marques and Queiroz (2012), Brasil }\end{array}$ & $\begin{array}{l}\text { Analisar o cuidado ao adolescente na } \\
\text { atenção básica sob a ótica desses } \\
\text { sujeitos, enfocando suas necessidades de } \\
\text { saúde e a interação com os trabalhadores } \\
\text { de saúde. }\end{array}$ & $\begin{array}{l}\text { Estudo qualitativo, do tipo descritivo, } \\
\text { com grupos focais com } 15 \text { adolescentes, } \\
\text { estudantes de uma escola pública } \\
\text { de Fortaleza-CE } \\
\text { Na análise dos dados foram agrupados } \\
\text { núcleos temáticos, conforme a técnica de } \\
\text { análise de conteúdo, da qual emergiram } \\
\text { as categorias Realizados } \\
\text { Período agosto e outubro de } 2009\end{array}$ & $\begin{array}{l}\text { Ocorreu grande insatisfação dos adolescentes em todos os } \\
\text { momentos da atenção, devido a problemas de comunicação, } \\
\text { atendimento impessoal, tempo insuficiente de consulta, falta } \\
\text { de vínculo e horizontalidade com os profissionais } \\
\text { Foi evidenciada uma falta de capacitação técnica profissional }\end{array}$ \\
\hline $\begin{array}{c}\text { Acolhimento à adolescente na consulta de } \\
\text { enfermagem - estudo qualitativo } \\
\text { Braz et al. (2013), Brasil }\end{array}$ & $\begin{array}{l}\text { Compreender a relação do enfermeiro } \\
\text { com a mãe adolescente na consulta de } \\
\text { puericultura sob o enfoque do } \\
\text { acolhimento }\end{array}$ & $\begin{array}{l}\text { Estudo qualitativo, realizado em uma } \\
\text { Unidade Básica de Saúde de Fortaleza- } \\
\text { CE, utilizando a observação sistemática } \\
\text { e a entrevista semiestruturada, com cinco } \\
\text { enfermeiros e três mães adolescentes na } \\
\text { consulta de puericultura, com análise de } \\
\text { conteúdo de Bardin, da qual emergiram } \\
\text { as categorias } \\
\text { Período de fevereiro a maio de } 2011\end{array}$ & $\begin{array}{l}\text { A importância da escuta de qualidade, de maneira cordial, } \\
\text { respeitosa e educada para preservar a individualidade e o } \\
\text { melhor amparo do adolescente } \\
\text { A atenção aos adolescentes deve basear-se na integralidade } \\
\text { As adolescentes reclamaram sobre os déficits no horário do } \\
\text { atendimento e que a consulta de puericultura sempre voltada } \\
\text { ao bebê, só que as enfermeiras alegaram a não percepção por } \\
\text { parte das adolescentes que muitas orientações eram } \\
\text { direcionadas para elas }\end{array}$ \\
\hline $\begin{array}{l}\text { The Perception of South African } \\
\text { Adolescents Regarding Primary Health } \\
\text { Care Services } \\
\text { Richter and Mfolo (2006), África do Sul }\end{array}$ & $\begin{array}{l}\text { Descrever as preferências dos } \\
\text { adolescentes em relação aos serviços de } \\
\text { atenção primária }\end{array}$ & $\begin{array}{l}\text { Pesquisa quanti-qualitativa descritiva, } \\
\text { transversal, com entrevistas } 119 \\
\text { adolescentes de uma região rural da } \\
\text { África do Sul } \\
\text { Análise dos dados quantitativos pelo }\end{array}$ & $\begin{array}{l}\text { Os adolescentes desejavam participar do planejamento das } \\
\text { atividades do serviço de saúde do adolescente } \\
\text { A simpatia e o respeito aos adolescentes eram características } \\
\text { desejáveis de um serviço de saúde amigo do adolescente; } \\
\text { preferiram profissionais jovens; horários flexíveis de }\end{array}$ \\
\hline
\end{tabular}


Research, Society and Development, v. 10, n. 2, e58710212936, 2021

(CC BY 4.0) | ISSN 2525-3409 | DOI: http://dx.doi.org/10.33448/rsd-v10i2.12936

\begin{tabular}{|c|c|c|c|}
\hline & & $\begin{array}{l}\text { SPSS e análise dos dados qualitativa das } \\
\text { questões abertas pela análise temática }\end{array}$ & $\begin{array}{l}\text { atendimento; centros de atendimentos próximos às suas } \\
\text { residências; acolhimento humanizado; diferentes profissionais } \\
\text { na equipe de saúde, especificidade ao público jovem e } \\
\text { separados dos serviços adultos }\end{array}$ \\
\hline $\begin{array}{l}\text { Health service utilization and reported } \\
\text { satisfaction among adolescents in Dejen } \\
\text { district, Ethiopia: a cross-sectional study } \\
\text { Dagnew et al. (2015), Etiópia }\end{array}$ & $\begin{array}{l}\text { Relatar a utilização do serviço de } \\
\text { saúde por adolescentes e sua satisfação } \\
\text { quanto ao seu funcionamento }\end{array}$ & $\begin{array}{l}\text { Estudo transversal, de base } \\
\text { comunitária, com entrevista realizadas } \\
\text { em } 690 \text { adolescentes, de } 15 \text { a } 19 \text { anos, no } \\
\text { distrito de Dejen, Etiópia, para verificar } \\
\text { satisfação por meio de escala likert } 3 \\
\text { pontos } \\
\text { Período } 5 \text { e } 17 \text { de fevereiro de } 2012\end{array}$ & $\begin{array}{l}\text { A utilização dos serviços de saúde foi baixa pelos } \\
\text { adolescesntes } \\
\text { Fatores que determinam o nível de satisfação dos } \\
\text { adolescentes: distância de casa ao Centro de Saúde, presença } \\
\text { de fármacos na unidade, recebimento do serviço desejado e } \\
\text { garantia da privacidade do jovem, principalmente } \\
\text { o relacionamento de longo prazo e confiável com profissionais } \\
\text { de saúde } \\
\text { A satisfação dos adolescentes com os serviços de saúde foi } \\
\text { baixa e determinada por vários fatores que estão relacionados } \\
\text { à acessibilidade, aceitabilidade do serviço de saúde e relação } \\
\text { paciente/profissional }\end{array}$ \\
\hline $\begin{array}{l}\text { What Teens Want: Barriers to Seeking } \\
\text { Care for Depression } \\
\text { Wisdom et al. (2006), Estados Unidos da } \\
\text { América }\end{array}$ & $\begin{array}{l}\text { Examinar as experiências de } \\
\text { adolescentes em busca ou em tratamento } \\
\text { para depressão dentro do setor primário } \\
\text { de serviços de saúde }\end{array}$ & $\begin{array}{l}\text { Entrevista por meio de grupo focal } \\
\text { pós-escola de } 90 \text { minutos com } 7 \\
\text { adolescentes de uma escola secundária } \\
\text { local } \\
\text { Washington, EUA }\end{array}$ & $\begin{array}{l}\text { A adesão e aceitação do tratamento proposto pelos } \\
\text { profissionais pelos adolescentes é efetiva quando eles se } \\
\text { sentem parte ativa da tomada de decisões sobre sua própria } \\
\text { saúde } \\
\text { É necessário incluí-los na tomada de decisões considerando } \\
\text { sua autonomia e individualidade } \\
\text { Quando os profissionais expressam empatia e trocam }\end{array}$ \\
\hline
\end{tabular}




\begin{tabular}{|c|c|c|c|}
\hline & & & $\begin{array}{l}\text { informações, os adolescentes se sentem mais conectados } \\
\text { A preocupação com a confidencialidade foi um fator } \\
\text { importante na disposição dos adolescentes em abordar um } \\
\text { profissional }\end{array}$ \\
\hline $\begin{array}{r}\begin{array}{r}\text { Trust, negotiation, and communication: } \\
\text { youngadults'experiences of primary care } \\
\text { services } \\
\text { Davey et al. (2013), Reino Unido }\end{array}\end{array}$ & $\begin{array}{l}\text { Descobrir os possíveis motivos para a } \\
\text { insatisfação dos adolescentes quanto ao } \\
\text { cuidado do serviço de saúde }\end{array}$ & $\begin{array}{l}\text { Estudo qualitativo com análise } \\
\text { temática de entrevistas semiestruturadas } \\
\text { de } 20 \text { jovens, explorando suas } \\
\text { experiências e uso de serviços de } \\
\text { atenção primária } \\
\text { Devon, Reino Unido }\end{array}$ & $\begin{array}{l}\text { O estudo ressalta a importância da confidencialidade do } \\
\text { atendimento, não julgamento e privacidade das informações } \\
\text { passadas pelos adolescentes aos profissionais, além da } \\
\text { expressão de empatia na comunicação; horários flexíveis e } \\
\text { localização mais conveniente } \\
\text { A confiança foi construída ao longo do tempo por meio da } \\
\text { continuidade dos cuidados } \\
\text { Houve falta de compreensão sobre seus direitos como } \\
\text { pacientes, uma vez que os jovens se tornam legalmente } \\
\text { responsáveis por seus próprios cuidados de saúde. } \\
\text { Os jovens mostraram-se insatisfeitos quando os pais foram } \\
\text { mais ouvidos do que eles próprios e quando não foi } \\
\text { estabelecido um vínculo de confiança com o profissional }\end{array}$ \\
\hline $\begin{array}{l}\text { Cirurgião-dentista ideal: perfil definido } \\
\text { por crianças e adolescentes } \\
\text { Bottan et al. (2009), Brasil }\end{array}$ & $\begin{array}{l}\text { Analisar a percepção de crianças e } \\
\text { adolescentes quanto ao dentista ideal }\end{array}$ & $\begin{array}{l}\text { Estudo descritivo, com coleta de } \\
\text { dados em } \\
\text { escolares do ensino fundamental }\left(5 .^{\mathrm{a}} \text { a }\right. \\
8 .^{\text {a }} \text { série) sobre a percepção do dentista } \\
\text { ideal } \\
\text { Análise dos dados: análise de }\end{array}$ & $\begin{array}{l}\text { O acolhimento humanizado e escuta do paciente de forma } \\
\text { qualificada dos profissionais tem um papel importante no } \\
\text { processo de cuidado para o grupo etário }\end{array}$ \\
\hline
\end{tabular}


Research, Society and Development, v. 10, n. 2, e58710212936, 2021

(CC B Y 4.0) | ISSN 2525-3409 | DOI: http://dx.doi.org/10.33448/rsd-v10i2.12936

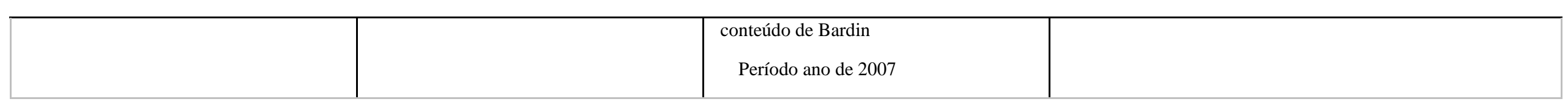

Fonte: Autores.

Após análise dos estudos foram identificadas condições nos serviços de saúde que interferem na atenção à saúde dos adolescentes. Essas condições foram classificadas segundo o contexto em que se apresentam como condições de dimensões macro, meso ou micro. A Figura 2 apresenta a ocorrência das dimensões observadas nos trabalhos analisados. 
Figura 2 - Condições nos serviços de saúde que interferem na atenção à saúde dos adolescentes: dimensões macro, meso e micro, de acordo com os artigos selecionados.

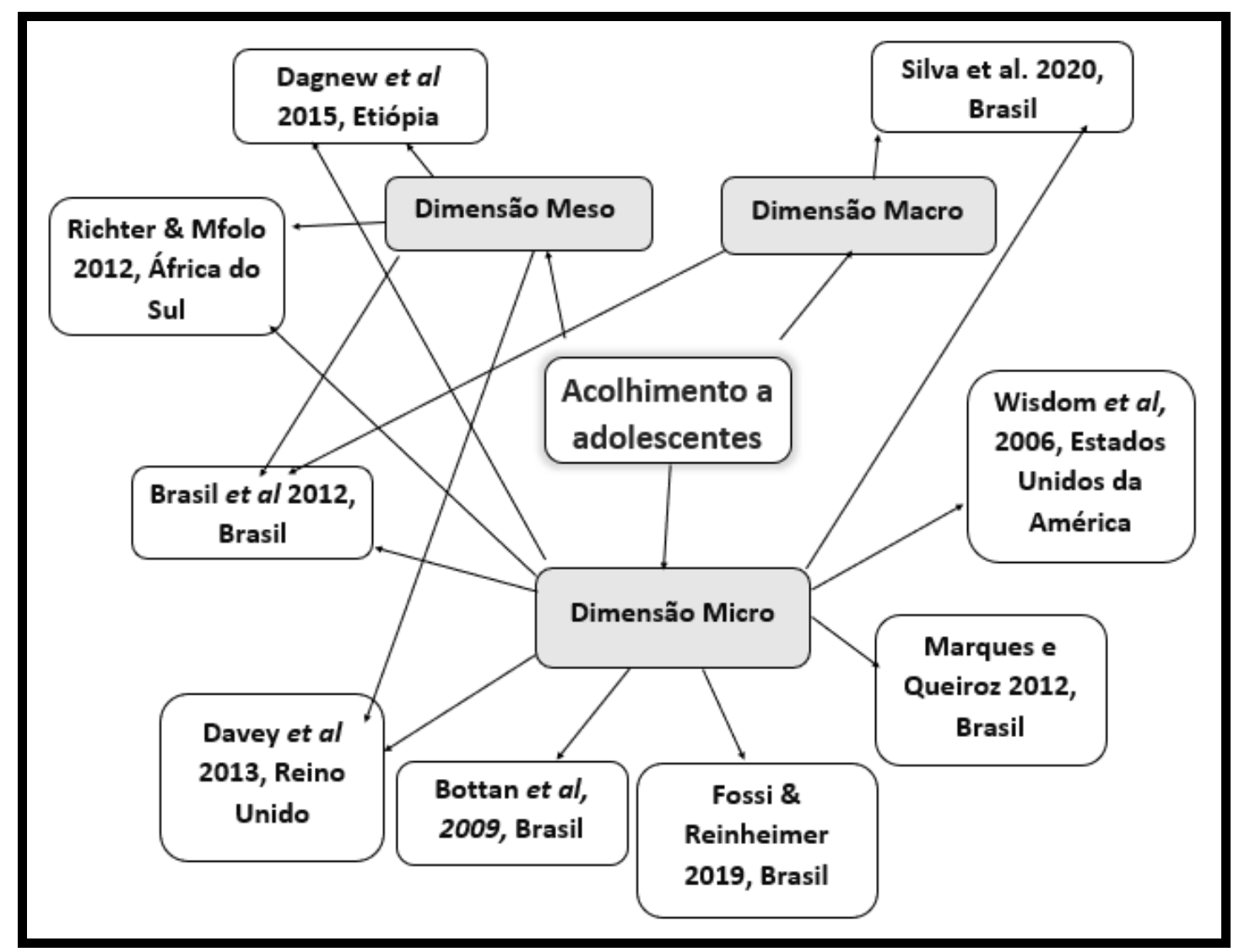

Fonte: Autores.

A dimensão macro refere-se ao modelo de atenção onde o adolescente é atendido prevalecendo características do modelo biomédico, com ausência do acompanhamento longitudinal. A dimensão meso se refere às condições estruturais e físicas dos centros de atendimentos e organização do processo de trabalho com horários de atendimentos limitados, tempo insuficiente de consulta, localização distante dos centros de atendimentos em relação à residência dos adolescentes, ausência de profissionais de saúde com perfil para o atendimento desse grupo populacional. Já a dimensão micro diz respeito às relações interpessoais entre os adolescentes e os profissionais de saúde com ausência dos vínculos de confiança; falta de empatia e simpatia da equipe de saúde, inexistência de uma relação horizontal entre o jovem e a equipe de saúde, falta de participação ativa do adolescente na tomada de decisões com ausência da escuta qualificada e falta de respeito com a privacidade do adolescente.

A Figura 3 apresenta de forma sintética os fatores que interferem nos cuidados à saúde dos adolescentes identificados nos trabalhos analisados. 
Figura 3. Fatores que interferem no acolhimento aos adolescentes identificados nos artigos selecionados nesta revisão.

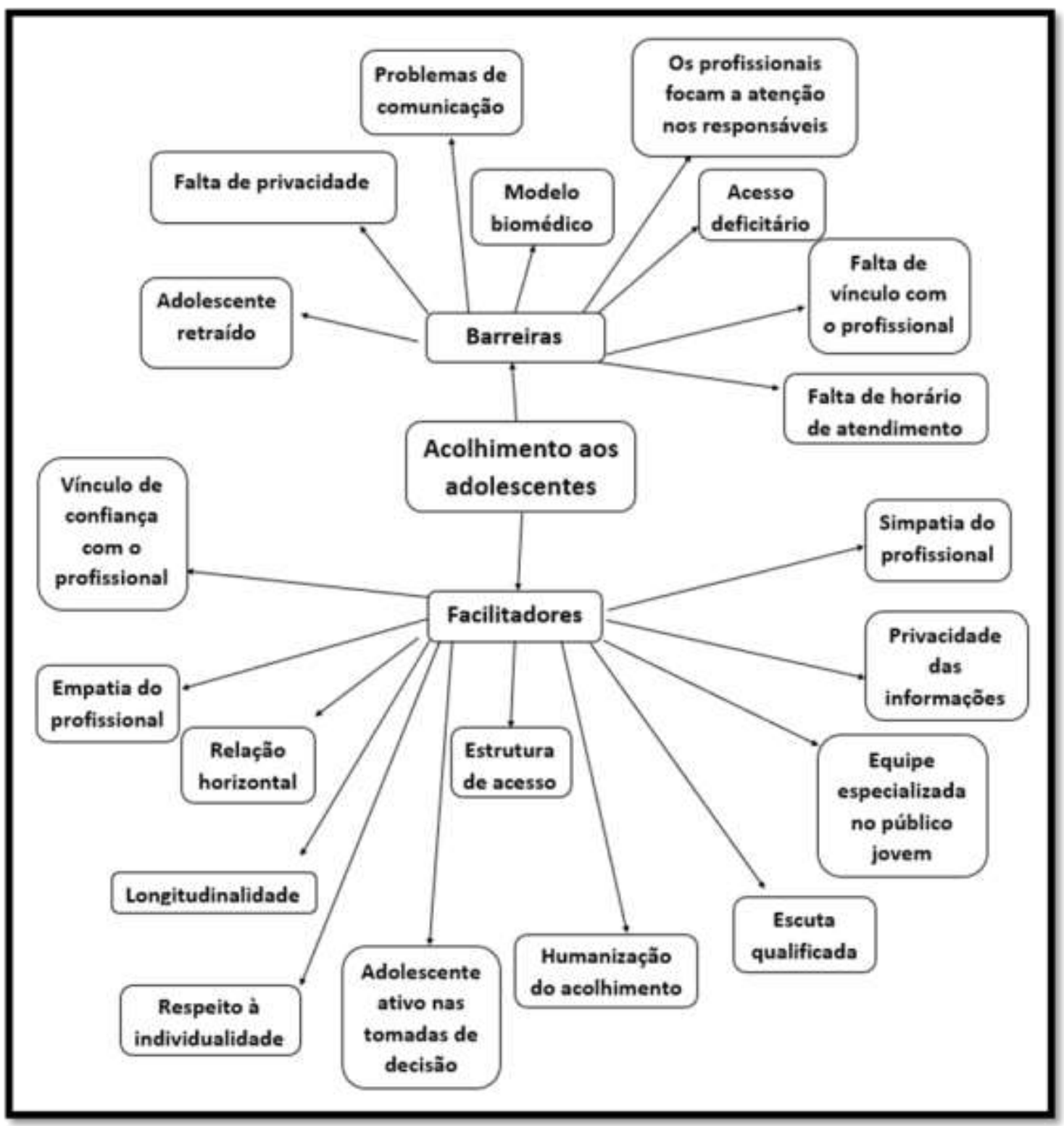

Fonte: Autores.

Para cada uma das dimensões, foram identificados facilitadores e barreiras para o acolhimento adequado e atenção à saúde dos adolescentes. Atendimento humanizado; acesso facilitado do adolescente; respeito a privacidade e inclusão na tomada de decisão; profissional jovem capacitado e empático ao atendimento do adolescente foram identificados como facilitadores do cuidado a este público. As barreiras apontadas foram a falta da humanização no atendimento, evidenciando o modelo centrado na doença (biomédico), com falhas na comunicação, horários de consultas não apropriados e não observação das particularidades da população adolescente.

\section{Discussão}

A pesquisa trouxe achados sobre fatores que influenciam no acolhimento e no acesso à atenção à saúde de adolescentes. O acolhimento como diretriz fundamental no estabelecimento de um modelo humanizado, estruturado para a atenção ao público adolescente, com foco no respeito as individualidades do usuário, com estabelecimento de uma escuta qualificada e constituição de vínculos de confiança, mostrou-se indispensável para a garantia da qualidade e longitudinalidade 
do cuidado.

Um estudo evidenciou uma baixa procura dos adolescentes pelos serviços de saúde (Dagnew et al., 2015). No Brasil, por meio da pesquisa nacional PENSE (IBGE, 2016), realizada com escolares do Brasil em 2015, mostrou que uma parcela aquém da totalidade (54,5\% dos escolares de 13 a 15 anos e 60,4\% de 16 a 17 anos) procurou o serviço de saúde, mostrando um dado preocupante levando em conta as necessidades e particularidades dessa fase da vida. Adolescentes e jovens, por serem considerados pessoas saudáveis, não são consideradas pessoas com necessária atenção à saúde, a não ser nas questões de saúde reprodutiva (Brasil, 2010). Infelizmente, há um entendimento que adolescentes raramente ficam doentes e o hábito de se justificar qualquer problema de saúde, seja físico ou mental, como comum ou característico dessa etapa da vida, limita a atenção à saúde dos adolescentes (Braz et al., 2013).

Nos trabalhos internacionais que abordam o atendimento ao adolescente, não foram encontrados estudos sobre perfis de atendimento que correspondessem em suas práticas ao que estabelece a Política Nacional de Humanização para o acolhimento. Assim sendo, os trabalhos foram categorizados nas dimensões de análise segundo seus achados quanto à acessibilidade e outras dimensões a serem observadas na atenção à saúde do adolescente.

Quanto à dimensão macro, os estudos mostraram que é necessário um modelo de atenção humanizado para o acolhimento e atenção à saúde do adolescente. O modelo de atenção à saúde ainda hegemônico está estruturado para a resolução de questões relacionados aos sintomas clínicos das doenças, com enfoque individual e dissociado do contexto social e emocional do indivíduo. Uma das consequências é a fragmentação da atenção à saúde e consequente ineficiência frente às necessidades da população (Silva et al., 2020) e a atenção aos adolescentes deve basear-se na integralidade da atenção (Brasil et al., 2012).

Essa fragmentação é elemento dificultador no atendimento dos adolescentes uma vez que essa população necessita de planos de cuidado diferenciados quanto ao envolvimento da equipe quanto na abordagem desses usuários, tanto na participação dos profissionais de saúde quanto na abordagem dos usuários. O modelo biomédico de atenção à saúde, com foco eminentemente curativo e ausência de uma abordagem humanizada e interpessoal podem criar barreiras ao acesso do adolescente ao sistema de saúde (Silva et al., 2020). Políticas públicas voltadas para a atenção à saúde do adolescente são importantes para redirecionar a atenção à saúde de acordo com as demandas e especificidades dos adolescentes.

Em relação à dimensão meso, pelas características próprias dos adolescentes, é possível que haja dificuldades em relação ao cumprimento de horários e ou datas de agendamento. Nesse caso faz-se necessário investir em ações educativas e com envolvimento efetivo da equipe de saúde, o que poderá sensibilizar essa população para sentir-se corresponsável para o cuidado com a própria saúde.

Serviços de saúde com acesso e acessibilidade para a população adolescente deve ser uma prioridade (Richter \& Mfolo, 2006).

A OMS preconiza que os centros de atendimento em saúde devam estar localizados de 5 a $10 \mathrm{~km}$ de distância da residência do usuário adolescente e o transporte deve ser de fácil acesso (WHO, 1986). Em pesquisa realizada por Dagnew et al. (2015), verificou-se que o grau de satisfação com o serviço de saúde estava diretamente relacionado com a distância dos Centros de Saúde. Dessa forma, adolescentes que tinham residência localizada nas proximidades dos locais de atendimento tiveram 3,6 vezes mais chances de estarem satisfeitos com os serviços de saúde em relação àqueles com residência mais distante.

Há necessidade de considerar a flexibilização dos horários de atendimento do adolescente uma vez essa idade é marcada pelo processo de independência em relação aos seus responsáveis. Nesse sentido, a busca pelos serviços de saúde também pode acontecer de forma independente (Davey et al., 2013). A dificuldade na marcação das consultas pode ser um dificultador no acesso de adolescentes aos sistemas de saúde e horários não flexíveis e incompatíveis com a real rotina dos 
jovens desestimulam a busca por atendimento. Além disso, outra barreira é a demora na oferta de oportunidades de consulta ocasionando desistência (Marques \& Queiroz, 2012; Richter \& Mfolo, 2006).

Entre os trabalhos relacionados neste estudo, foi apontado que a satisfação pelos serviços de saúde aumenta quando estão disponíveis condições de infraestrutura para atendimento exclusivo aos adolescentes. A separação de atendimento dessa população dos adultos confere aos jovens a autonomia, já que muitos preferem não ter a supervisão dos responsáveis nessas ocasiões (Richter \& Mfolo, 2006). A construção de uma relação satisfatória pode ser favorecida com o uso da estrutura da recepção para o desenvolvimento de atividades, principalmente as de educação em saúde (Emmi et al., 2016). A educação em saúde é fortemente sensível na abordagem da saúde do adolescente que necessita de informações oportunas e confiáveis acerca de comportamentos próprios dessa faixa etária que apresentam risco para saúde (Richter \& Mfolo, 2006). Informações sobre serviços prestados pelas unidades de saúde devem ser disponibilizadas, evitando, desse modo, que os jovens, por falta de conhecimento, busquem atendimento à saúde incompatíveis com as suas necessidades (Davey et al., 2013).

Nos serviços de saúde é necessário que os profissionais sejam engajados e capacitados para atuação junto aos jovens, atentos as nuances que permeiam as relações interpessoais. Nesse sentido, é fundamental o trabalho em equipe para garantir a longitudinalidade e eficiência do tratamento ao adolescente (Marques \& Queiroz, 2012).

Dois componentes que podem determinar uma atenção à saúde do adolescente com qualidade é a participação da equipe no planejamento dessas ações e o investimento na educação permanente como espaço de formação segundo as necessidades de cuidado dessa população (Marques \& Queiroz, 2012).

A ausência de investimento no cuidado interprofissional e na interlocução na equipe favorecem o abandono do cuidado por parte do paciente (Lobo et al., 2020; Silva et al., 2020). Nesse sentido, pode-se prever que esse impacto será mais expressivo entre os adolescentes considerando as especificidades que marcam essa etapa de vida.

Estudo de Richter and Mfolo (2006) demonstrou que um fator determinante para a acessibilidade de adolescentes aos serviços de saúde é capacidade financeira dessa população em arcar com os custos resultantes do atendimento. Corroborando com esse estudo, Dagnew et al. (2015) constataram que adolescentes tiveram acesso gratuito aos medicamentos no serviço público de saúde, apresentavam 2,7 vezes mais chances de estarem satisfeitos com a prestação de serviços.

Em relação à dimensão micro, as relações interpessoais, a aceitação e ingresso efetivo de adolescentes no sistema de saúde estão diretamente ligados à interação estabelecida entre os pacientes e os profissionais. O modo como o profissional trata e interage com o paciente é geralmente mais importante para o adolescente, antes mesmo de características relacionadas à profissionalidade, como competência, experiência e pontualidade (Bottan et al., 2009). Quando as opiniões e desejos do adolescente não são levadas em consideração na tomada de decisões sobre a sua saúde, a insatisfação com o serviço de saúde é muito maior, assim como o abandono dos tratamentos propostos (Wisdom et al., 2006).

Muitos adolescentes apresentam dificuldades em compartilhar suas questões pessoais e necessidades com a equipe de saúde, principalmente quando estão acompanhados por seus responsáveis (Richter \& Mfolo, 2006). As informações sigilosas que, se propagadas, podem gerar consequências negativas para o adolescente (Davey et al., 2013; Wisdom et al., 2006) devem ser protegidas pela confidencialidade. Esse é um fator determinante para a satisfação dos jovens com o serviço de saúde. Dagnew et al. (2015) demonstraram que quando os adolescentes tiveram sua privacidade preservada no atendimento, sua satisfação foi de 2,9 vezes maior.

Quanto à insatisfação, esta fica demonstrada quando os profissionais de saúde se importam mais em escutar os responsáveis do que o jovem, o que reforça a expectativa de que os responsáveis não estejam presentes na consulta. Entretanto, o envolvimento e orientação dos responsáveis pode ser benéfica para o atendimento, porém não cerceando a liberdade e autonomia dos jovens na tomada de decisão sobre sua própria saúde (Wisdom et al., 2006).

Frequentemente os adolescentes são tratados como sujeitos não autônomos, porém é esperado que eles tenham 
responsabilidade pelos seus atos em relação à própria saúde. Isso demonstra uma incoerência na forma de como essa população é percebida (Marques \& Queiroz, 2012). Nesse sentido, é necessário que os responsáveis pelos adolescentes e a equipe de saúde valorizem a autonomia dessa população para que ela se sinta corresponsável pelo cuidado com a sua saúde $\mathrm{O}$ envolvimento do adolescente no planejamento das atividades relacionadas à saúde contribui para que os serviços se tornem mais acessíveis e atraentes às necessidades particulares desse público (WHO, 1986).

Os pais devem incentivar que seus filhos busquem participar das campanhas de saúde, depositando uma visão encorajadora e positiva sobre os serviços, já que existe uma relutância dos jovens em buscar por atendimento (Fossi \& Reinheimer, 2019; Richter \& Mfolo, 2006). Essa dificuldade pode ser explicada pela grande cobrança que o adolescente coloca sobre si uma vez que a busca por atendimento pode representar uma fragilidade tornando-se controversa a expectativas que ele tem sobre si mesmo (Wisdom et al., 2006).

O interesse do paciente pelo atendimento está diretamente relacionado com o vínculo de confiança estabelecido com a equipe de saúde (Davey et al., 2013; Marques \& Queiroz, 2012; Silva et al., 2020). O vínculo será maior na presença de uma escuta qualificada que leve em consideração os receios e desejos do adolescente, assim como a confidencialidade (Davey et al., 2013). O respeito, tolerância, disposição, paciência, acessibilidade, e empatia são elementos necessários para que o paciente se sinta acolhido e com o sentimento de confiança em relação à equipe (Bottan et al., 2009; Richter \& Mfolo, 2006). A escuta deve observar a compreensão do contexto de vida de cada paciente e o profissional deve se mostrar sensível às informações trazidas pelo adolescente (Brasil et al., 2012; Wisdom et al., 2006). Os resultados do estudo de Dagnew et al. (2015) mostraram que os pacientes que foram acolhidos por atendentes respeitosos e amigáveis e tiveram uma comunicação satisfatória com os profissionais, apresentaram uma satisfação com o atendimento 3 vezes maior do que aqueles que não tiveram uma boa experiência no campo relacional. Uma comunicação eficiente entre adolescente e profissional de saúde, com uso de uma linguagem clara, é indispensável para que as ações de cuidado à saúde alcancem bons resultados (Brasil et al., 2012; Wisdom et al., 2006).

A abordagem identificada nesta revisão sobre o acolhimento e atenção à saúde aos adolescentes pode servir para direcionar e embasar ações de organização e planejamento em serviços de saúde que atendem esta parcela populacional.

Como limitação deste estudo pode-se apontar a necessidade da ampliação e diversificação das bases de dados consultadas com maior aporte de literatura internacional.

\section{Considerações Finais}

Os resultados deste estudo sugerem que o atendimento à saúde do adolescente exige iniciativas que respondam às especificidades dessa população.

O acolhimento é fundamental para aproximar o adolescente dos cuidados em saúde, pois humaniza e otimiza o atendimento, cria vínculo, respeito pela individualidade e autonomia.

Dos serviços de saúde espera-se o investimento em programas específicos, equipamentos com capacidade resolutiva e postura da equipe atenta aos componentes relacionais do processo de cuidado à saúde.

A escassa literatura aponta a necessidade de realização de estudos futuros sobre o acolhimento e atenção à saúde aos adolescentes, com metodologias mais robustas, bem delineadas, de natureza quantitativa e qualitativa.

\section{Agradecimentos}

Á Pró-Reitoria de Pesquisa da UFMG pelo Programa Institucional de Auxílio à Pesquisa de Docentes RecémContratados e bolsista de Iniciação Científica da UFMG. 


\section{Referências}

Bottan, E. R., Oglio, J. D., Garcia, E. S., \& Araújo, S. M. (2009). Cirurgião-dentista ideal: perfil definido por crianças e adolescentes. Revista Sul-Brasileira de Odontologia 6(4), 381-386.

Brasil. (2004). HumanizaSUS: Política Nacional de Humanização: a humanização como eixo norteador das práticas de atenção e gestão em todas as instâncias do SUS. Editora do Ministério da Saúde.

Brasil. (2010). Acolhimento nas práticas de produção de saúde. Editora do Ministério da Saúde.

Brasil. (2017). Proteger e cuidar da saúde de adolescentes na atenção básica. Editora do Ministério da Saúde.

Brasil, E. G. M., Queiroz, M. V. O., \& Cunha, J. M. H. (2012). Receptiveness to the teenager in nursing consultation - descriptive study Online Brazilian Journal of Nursing, 11(2), 346-358.

Braz, M., Filho, A. A. B., \& Barros, M. B. A. (2013). Saúde dos adolescentes: um estudo de base populacional em Campinas, São Paulo, Brasil. Cadernos de Saúde Pública, 29(9), 1877-1888.

Dagnew, T., Tessema, F., \& Hiko, D. (2015). Health Service Utilization and Reported Satisfaction among Adolescents in Dejen District, Ethiopia: A CrossSectional Study. Ethiopian Journal of Health Sciences, 25(1), 17-28.

Davey, A., Asprey, A., Carter, M., \& Campbell, J. L. (2013). Trust, negotiation, and communication: young adults' experiences of primary care services. BMC Fam Pract, 14, 202.

Emmi, D. T., Gomes, J. T., Barroso, R. F. F., \& Araújo, M. V. d. A. (2016). Humanização no acolhimento aos usuários das clínicas de ensino da Faculdade de Odontologia da Universidade Federal do Pará: cinco anos de experiência de um projeto de extensão. Revista Conexão UEPG, 12(3), 476-486.

Fossi, L. B., \& Reinheimer, F. K. (2019). Acolhimento coletivo como espaço de cuidado: uma análise das demandas em saúde mental na adolescência. Revista Psicologia Saúde 11(3), 35-48.

Grant, M. J., \& Booth, A. (2009). A typology of reviews: an analysis of 14 review types and associated methodologies. Health Info Libr J, 26(2), 91-108.

Hennington, É. A. (2005). Acolhimento como prática interdisciplinar num programa de extensão universitária. Cadernos de Saúde Pública, 21(1), 256-265. IBGE. (2016). Pesquisa nacional de saúde do escolar: 2015

Lobo, B. M. I. D. S. L., Xavier, S. C. M., Cortez, E. A., Mesquita, L. M., Valente, G. S. C., \& Soeiro, R. d. L. (2020). Strategies used by health professionals to accommodate the nursing mother: an integrative review. Research, Society and Development, 9(8), e13985168.

Marques, J. F., \& Queiroz, M. V. O. (2012). Cuidado ao adolescente na atenção básica: necessidades dos usuários e sua relação com o serviço. Revista Gaúcha Enfermagem, 33(3), 65-72.

Richter, M. S., \& Mfolo, V. (2006). The Perception of South African Adolescents Regarding Primary Health Care Services. The Scientific World Journal, 6, 737-744.

Rother, E. T. (2007). Revisão sistemática x revisão narrativa. Acta Paulista de Enfermagem, 20(2), v-vi.

Silva, B. G. d. A., Machado, A. N., Nóbrega, V. M., Oliveira, R. C., Vaz, E. M. C., \& Collet, N. (2020). Gestão do cuidado à criança/adolescente /adolescentecom doença crônica: (des)articulação com doença crônica: (des)articulação da rede e fragmentação das ações. Revista de Enfermagem UFSM $10(\mathrm{e} 76), 1-21$.

WHO. (1986). Young people's health-a challenge for society. Report of a WHO Study Group on young people and "Health for All by the Year 2000". World Health Organ Tech Rep Ser, 731, 1-117.

Wisdom, J. P., Clarke, G. N., \& Green, C. A. (2006). What teens want: barriers to seeking care for depression. Administration policy mental health 33(2), 133145. 